\title{
ANALYSIS OF A SEMILINEAR PDE FOR MODELING STATIC SOLUTIONS OF JOSEPHSON JUNCTIONS*
}

\author{
J.-G. CAPUTO ${ }^{\dagger}$, N. FLYTZANIS $\ddagger$, A. TERSENOV , AND E. VAVALIS $^{\S}$
}

\begin{abstract}
A semilinear elliptic partial differential equation problem that models the static (zero voltage) behavior of a Josephson window junction is considered. A priori estimates and differential properties of the solution are obtained. The existence of the solutions is shown and iterative methods for solving this problem are analyzed. Experimental numerical data that couple with the theoretical results are presented. Useful physical information is extracted from our analysis
\end{abstract}

Key words. semilinear elliptic equations, a priori estimates, Josephson window junction

AMS subject classifications. 35J25, 35Q53

PII. S0036141002303673

1. Introduction. A Josephson junction is a weak link between two superconducting films separated by a thin oxide layer enabling the tunneling of Cooper pairs of electrons. The steady state operation under the action of an external magnetic field and bias with a constant external current is described by a semilinear elliptic partial differential equation (PDE) with a sinusoidal nonlinearity which arises from the Josephson tunneling current. The quantity that completely describes the electromagnetic properties of such a device is the difference $\phi(x, y)$ of the phases of the superconducting order parameters in the two films. The response of the junction to an external current and magnetic field depends crucially on the ratio of the junction dimensions $\mathcal{L}$ (length) and $\mathcal{W}$ (width) to the characteristic length of the problem, the Josephson penetration depth $\lambda_{j}$. Short junctions for which $\mathcal{L}, \mathcal{W}<\lambda_{j}$ are widely used in the static case (zero voltage) for magnetic field detection. When $\phi$ becomes time dependent the governing equation is of hyperbolic type, and such small junctions are used for voltage standard, while long junctions $\left(\mathcal{L}>\lambda_{j}>\mathcal{W}\right)$ are very high frequency oscillators $(>100 \mathrm{GHz})$ used in astrophysical measurements. An in-depth presentation of the physics and the technological applications of Josephson junctions can be found in [2].

The main difficulty of the resonant fluxon operation of a long junction is its low energy output compounded by a strong impedance mismatch at the boundaries. The coupling of the Josephson junction to a cavity in the so-called window design allows a better impedance matching $[6,4]$. It is also interesting for tailoring the static or zero voltage behavior of the device for specific purposes [10] like increasing the maximum allowed bias current in the absence of magnetic field. An extension of this model to inhomogeneous critical current density can be relevant for high $T_{c}$ superconducting materials with grain boundaries [7]. Finally static solutions can be considered as fixed points and play an important role in computing the solutions of the associated

* Received by the editors March 13, 2002; accepted for publication (in revised form) July 24, 2002; published electronically May 12, 2003. This work was supported in part by a French-Greek collaboration agreement and PENED grant 2028.

http://www.siam.org/journals/sima/34-6/30367.html

${ }^{\dagger}$ Laboratoire de Mathématiques, Institut de Sciences Appliquées, B.P. 8, 76131 Mont-SaintAignan cedex, France.

${ }^{\ddagger}$ Physics Department, University of Crete, 71409 Heraklion, Greece.

$\S$ Mathematics Department, University of Crete, 71409 Heraklion, Greece (tersenov@math.uoc.gr, mav@math.uoc.gr). 
hyperbolic time-dependent problem. In [4] we proposed a semilinear PDE problem which accurately and effectively modeled the static behavior of a window Josephson junction. This model enabled us to predict specific effects depending on the size of the cavity, such as the rescaling of $\lambda_{j}$ and the increase of the maximum current for zero magnetic field [5].

If an annular geometry is considered, the periodic boundary conditions are appropriate and in this case an exhaustive classification and stability analysis of the solutions has already been carried out in one and two space dimensions including time [8]. For this geometry one is limited only to solutions with integer number of fluxons. Note, however, that this eliminates many of the interesting physical solutions that arise due to the finite size and the possibility of continuously introducing flux from the boundaries as we vary the magnetic field $H$.

The static two-dimensional Josephson junction problem was solved numerically by Barone et al. [1] only in the homogeneous junction case by introducing a damping term. This transforms the equation into a semilinear diffusion equation which can be discretized using explicit finite differences. A careful choice of the initial condition can lead to stable static solutions, but this cannot be guaranteed in general. In particular the junction with inhomogeneous properties requires special care. In all cases the multiplicity of solutions makes the choice of initial conditions very important, so that we need to address the static problem directly. Notice also that both the proof of the existence of a solution and some regularity estimates can be obtained easily in this time-dependent case but that these results cannot be extended to the static limit, which turns out to be a more difficult problem.

The derivation of this PDE model together with preliminary numerical experiments was presented in [4] and is briefly discussed in section 2, where comments on several mathematical peculiarities inherent in our problem are also included. In particular the periodic nonlinear right-hand side and the Neumann boundary conditions lead to an obvious nonuniqueness of the solution. Note also that the coefficients of the operator are nonsmooth. Using the additional variable method proposed in [11] and $[17,18]$, we first obtain a priori estimates on the gradient of the solution that are of physical interest. We then prove, under certain assumptions, the existence and uniqueness of the solution and the convergence of a fixed point linearization method. The study of the stability of the solutions is under way and will not be considered here. In particular we obtain in section 3 a priori estimates of the gradient of the solution of $\phi$ and show that the gradients are Hölder continuous functions. Based on these estimates we prove in section 4 the existence of the solution and show that a generalized second derivative exists in $L_{2}$. Assuming that the domain is narrow enough, we show, in the case of zero Neumann boundary conditions in one direction, that the solution does not depend on the associated variable. In section 5 we obtain additional estimates for the solution and its first derivatives only in terms of the external current and the magnetic field applied to it. Furthermore, assuming that the solution is in a given interval, we improve our a priori estimates. In section 6 we prove the convergence of an iterative method for linearizing the semilinear PDE problem. Numerical results that couple with our theoretical results are presented in section 7, which also discusses their physical relevance. Our conclusions are given in section 8 .

2. The mathematical Josephson window junction model. Figure 2.1 shows a window junction for the case where the window $\Omega_{j}$ is a rectangle of size $\ell \times w$ centered in $\Omega$. The spatial variation of the difference $\phi$ of the superconducting phases in both superconductors is modeled accurately in the case where the surface inductances 


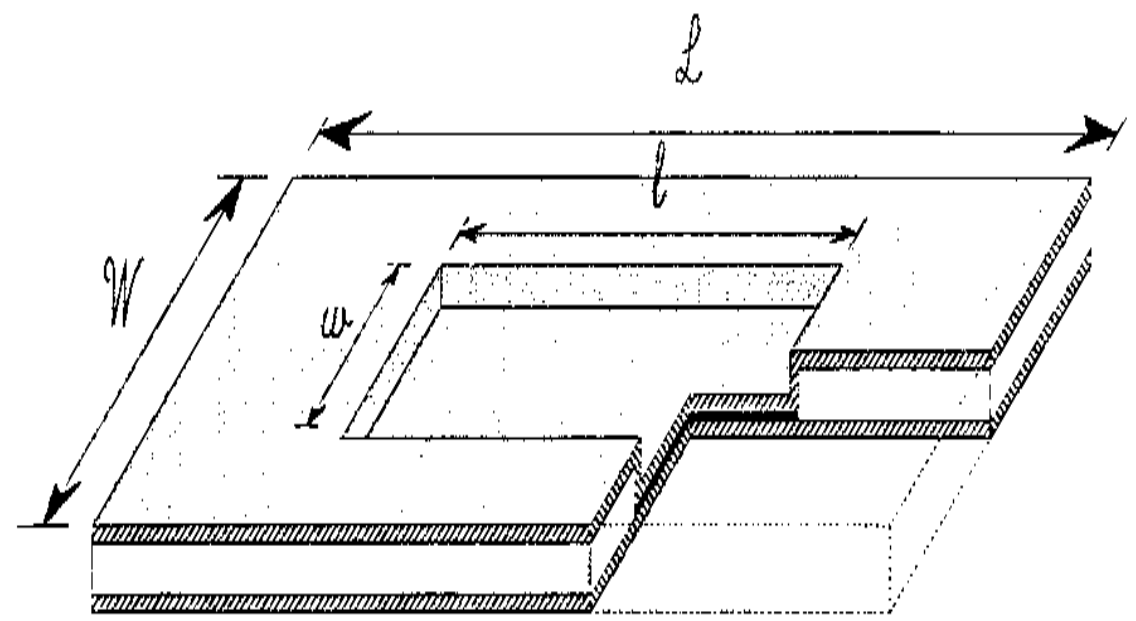

FIG. 2.1. A window Josephson junction.

are equal in the junction and the idle region by the equation

$$
\frac{\partial^{2} \phi}{\partial x^{2}}+\frac{\partial^{2} \phi}{\partial y^{2}}=\mathcal{I}_{j}(x, y) \sin (\phi) \text { in } \Omega \equiv\left(-\frac{\mathcal{L}}{2}, \frac{\mathcal{L}}{2}\right) \times\left(-\frac{\mathcal{W}}{2}, \frac{\mathcal{W}}{2}\right)
$$

coupled with the boundary conditions

$$
\left.\frac{\partial \phi}{\partial x}\right|_{x=-\frac{\mathcal{L}}{2}}=H-\alpha_{1},\left.\quad \frac{\partial \phi}{\partial x}\right|_{x=\frac{\mathcal{L}}{2}}=H+\alpha_{2},\left.\quad \frac{\partial \phi}{\partial y}\right|_{y=-\frac{\mathcal{W}}{2}}=-\delta_{1},\left.\quad \frac{\partial \phi}{\partial y}\right|_{y=\frac{\mathcal{W}}{2}}=\delta_{2}
$$

where all lengths have been normalized by $\lambda_{j}$. Physically $\mathcal{I}_{j}$ in $(2.1)$ is the indicator function of the domain $\Omega_{j}$ and is discontinuous. Although in the derivation of the results that will follow we have assumed that $\mathcal{I}_{j}$ is continuous, we will see that all our results are independent of the smoothness of $\mathcal{I}_{j}$.

The model given in (2.1) can be made more realistic by including the difference in the surface inductances in the superconducting and junction regions, which leads to the equation

$$
\frac{\partial}{\partial x}\left(\frac{1}{\tilde{L}(x, y)} \frac{\partial \phi}{\partial x}\right)+\frac{\partial}{\partial y}\left(\frac{1}{\tilde{L}(x, y)} \frac{\partial \phi}{\partial y}\right)=\mathcal{I}_{j}(x, y) \sin (\phi),
$$

where $\tilde{L}$ is the normalized surface inductance. We believe that the analysis presented below can be extended to cover the case where $\tilde{L}(x, y)$ is strictly positive and differentiable.

The boundness of the right-hand side of (2.1) determines the maximum allowed values for $\alpha_{1}, \alpha_{2}, \delta_{1}$, and $\delta_{2}$. To see this, integrate both sides of (2.1) and use Green's theorem to obtain

$$
\int_{\Omega_{j}} \sin \phi d x d y=\int_{\Omega} \nabla(\nabla \phi) d x d y=\int_{\partial \Omega} \frac{\partial \phi}{\partial n} d s=\left(\alpha_{1}+\alpha_{2}\right) \mathcal{W}+\left(\delta_{1}+\delta_{2}\right) \mathcal{L},
$$

from which we have that

$$
\left|\left(\alpha_{1}+\alpha_{2}\right) \mathcal{W}+\left(\delta_{1}+\delta_{2}\right) \mathcal{L}\right| \leq \mu\left(\Omega_{j}\right)
$$




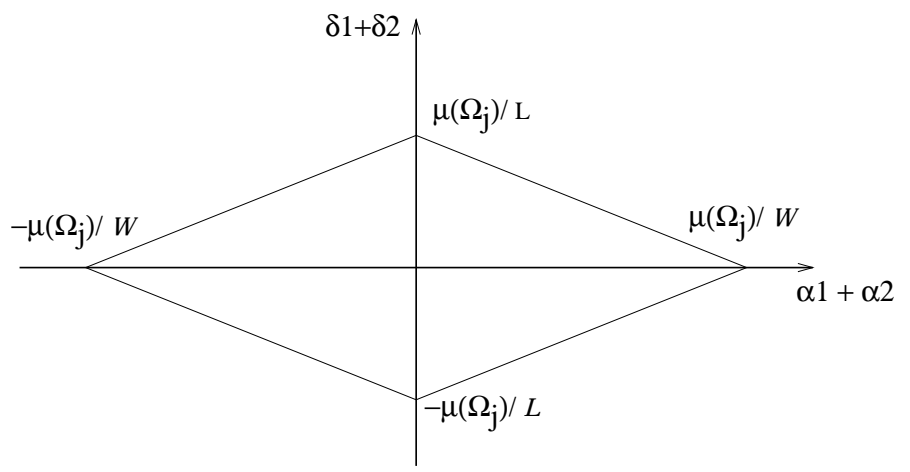

FIG. 2.2. Allowed values for $\alpha$ 's and $\delta$ 's.

where $\mu\left(\Omega_{j}\right)$ is the measure of the window domain $\Omega_{j}$. From (2.3) we easily see that our PDE problem has no solutions if the $\alpha$ 's and $\delta$ 's are outside the rhombus shown in Figure 2.2.

Physically $H$ corresponds to an external magnetic field applied in the $y$-direction which induces a gradient of $\phi$ along the $x$-direction. $\alpha$ 's and $\delta$ 's are current densities flowing through the device along the $x$ - and $y$-directions, respectively. They can be assumed to be positive and constant. As can be seen in Figure 2.2 the sum of these currents cannot exceed the maximum critical current of the junction, which is the measure of $\Omega_{j}$.

Notice also that if $\phi$ is a solution of the problem, then $\phi+2 k \pi, k \in Z$, is also a solution. This defines an equivalence class, so that solutions can be classified in terms of their fluxon content $n_{f \ell}$ defined by

$$
n_{f \ell} \equiv\left(\sup _{\Omega} \phi-\inf _{\Omega} \phi\right) /(2 \pi) .
$$

We also define the oscillation of $\phi(x, y)$ with respect to the variable $x$ (and similarly for the variable $y$ ) as

$$
o s c_{x} \phi \equiv \sup _{y}\left(\sup _{x} \phi-\inf _{x} \phi\right) .
$$

Depending on the boundary conditions we can have (see [4]) a one-fluxon solution where the oscillation is between 0 and $2 \pi$, a two-fluxon solution where the oscillation is between 0 and $4 \pi$, and so on. These different solutions will have different regions of existence and different stability properties with respect to a perturbation of the boundary conditions, and as the current is increased only one will subsist. This solution gives the maximum current at zero voltage of the junction, which can be observed experimentally to indicate the quality of the junction. In the inline configuration $\alpha_{1}=\alpha_{2}=\alpha, \delta_{1}=\delta_{2}=0$, in the absence of an idle region $\left(\Omega_{j}=\Omega\right)$, Owen and Scalapino showed that the maximum current for $H=0$ is $4 \mathcal{W}$ [15]. For that they reduced the problem to one dimension and wrote the solution in terms of elliptic functions. In the same geometry but with the overlap design for which $\alpha_{1}=\alpha_{2}=0$ $\delta_{1}=\delta_{2}=\delta$, the problem can be reduced to a one-dimensional equation only for $\mathcal{W}<2$ [3], yielding a maximum current for $H=0$ of $\mathcal{L} \times \mathcal{W}$. When $\mathcal{W}>2$ the current for $H=0$ saturates, as expected, to $4 \times \mathcal{L}$, and transverse modes are needed 
for the description [3]. The presence of an idle region $\left(\Omega_{j} \neq \Omega\right)$ has important effects on the behavior of the junction. In particular the characteristic length is larger than $\lambda_{J} \equiv 1$, which leads to an increase of the maximum current for $H=0$.

An important case is when the device is symmetric with respect to the center. Then if $\delta_{1}=\delta_{2}$, one can assume the solution to be symmetric with respect to the horizontal middle line, and if the $x$ boundary conditions are antisymmetric, i.e., $\alpha_{1}=$ $\alpha_{2}$ and $H=0$, the solution will be symmetric with respect to the vertical middle line, so that just a quarter of the device might be considered. A priori estimates have been derived for these cases also.

Notice also that the solution of the PDE problem is a minimum of the free energy functional

$$
\begin{aligned}
F= & \left.\int_{\Omega}\left[\frac{1}{2}\left(\frac{\partial \phi}{\partial x}\right)^{2}+\frac{1}{2}\left(\frac{\partial \phi}{\partial y}\right)^{2}+\mathcal{I}_{j}(1-\cos \phi)\right)\right] d x d y \\
& -\int_{-\frac{\mathcal{W}}{2}}^{\frac{\mathcal{W}}{2}}\left[\left(H-\alpha_{1}\right) \phi\left(-\frac{\mathcal{L}}{2}, y\right)-\left(H+\alpha_{2}\right) \phi\left(\frac{\mathcal{L}}{2}, y\right)\right] d y \\
& -\int_{-\frac{\mathcal{L}}{2}}^{\frac{\mathcal{L}}{2}}\left[-\delta_{2} \phi\left(x, \frac{\mathcal{W}}{2}\right)-\delta_{1} \phi\left(x,-\frac{\mathcal{W}}{2}\right)\right] d x
\end{aligned}
$$

Due to the multiplicity of solutions there are several minima. In the simple case where the boundary conditions are nonzero only in $x$ or $y$ and $\Omega \equiv \Omega_{j}$, the $y$ or $x$ dependence can be neglected and the last term of the free energy can be significantly simplified.

3. A priori estimates of the gradient. The main objective of this section is to obtain estimates of the gradient of the solution that are of practical interest in either proving the existence of the solution or measuring the gradient in terms of physical quantities. Estimates of the gradient in terms of the maximum of the solution are easily obtained from well-known results $[13,9]$. In this section we obtain a priori estimates for the gradient of a classical solution of the proposed PDE model only in terms of the size of the domain and the physical parameters of the problem. Note that the estimates obtained below are independent of the solution and cannot be obtained from classical results $[13,9]$.

We start by homogenizing the problem (2.1)-(2.2) by setting $u \equiv \phi-f$ with

$$
f \equiv H x+\frac{\alpha_{1}}{2 \mathcal{L}}\left(x-\frac{\mathcal{L}}{2}\right)^{2}+\frac{\alpha_{2}}{2 \mathcal{L}}\left(x+\frac{\mathcal{L}}{2}\right)^{2}+\frac{\delta_{1}}{2 \mathcal{W}}\left(y-\frac{\mathcal{W}}{2}\right)^{2}+\frac{\delta_{2}}{2 \mathcal{W}}\left(y+\frac{\mathcal{W}}{2}\right)^{2}
$$

to get from (2.1) and (2.2) that

$$
\frac{\partial^{2} u}{\partial x^{2}}+\frac{\partial^{2} u}{\partial y^{2}}=\mathcal{I}_{j} \sin (u+f)-\frac{\alpha_{1}+\alpha_{2}}{\mathcal{L}}-\frac{\delta_{1}+\delta_{2}}{\mathcal{W}} \text { in } \Omega
$$

and

$$
\left.\frac{\partial u}{\partial x}\right|_{x= \pm \frac{\mathcal{L}}{2}}=\left.\frac{\partial u}{\partial y}\right|_{y= \pm \frac{\mathcal{W}}{2}}=0 .
$$

In what follows, without explicitly stating, we assume that the indicator function $\mathcal{I}_{j}$ is smooth. This assumption is set only to guarantee the existence of a classical solution of the problem and does not affect the result of the lemmas since we do 
not have any smoothness requirements. In practice these indicator functions are discontinuous (i.e., $\mathcal{I}_{j}(x, y)$ is 1 if $(x, y) \in \bar{\Omega}_{j}$ and 0 otherwise). To treat such $\mathcal{I}_{j}$ we can consider a continuously differentiable function $\mathcal{I}_{j}^{\delta} \in C^{1}(\bar{\Omega}), 0 \leq \mathcal{I}_{j}^{\delta} \leq 1$, such that $\mathcal{I}_{j}^{\delta} \rightarrow \mathcal{I}_{j}{ }^{*}$ weak in $L_{\infty}$ for which the analysis that will follow is valid. Therefore in what follows and for simplicity in the notation we will use the symbol $\mathcal{I}_{j}$ instead of $\mathcal{I}_{j}^{\delta}$.

Lemma 3.1. For any classical solution $u(x, y)$ of the problem (3.1)-(3.2) we have that

$$
\left|u_{x}\right| \leq \mathcal{L}, \quad\left|u_{y}\right| \leq \mathcal{W}
$$

Proof. We start by writing (3.1) at a point $(\xi, y) \in \Omega$ with $\xi \neq x$ as

$$
\frac{\partial^{2} u(\xi, y)}{\partial \xi^{2}}+\frac{\partial^{2} u(\xi, y)}{\partial y^{2}}=\mathcal{I}_{j}(\xi, y) \sin (u(\xi, y)+f(\xi, y))-\frac{\alpha_{1}+\alpha_{2}}{\mathcal{L}}-\frac{\delta_{1}+\delta_{2}}{\mathcal{W}}
$$

Now define the function $v(x, y, \xi) \equiv u(x, y)-u(\xi, y)$, for which, by subtracting (3.4) from (3.1), we have

$$
\frac{\partial^{2} v}{\partial x^{2}}+\frac{\partial^{2} v}{\partial y^{2}}+\frac{\partial^{2} v}{\partial \xi^{2}}=\mathcal{I}_{j}(x, y) \sin (u(x, y)+f(x, y))-\mathcal{I}_{j}(\xi, y) \sin (u(\xi, y)+f(\xi, y)),
$$

and thus

$$
\frac{\partial^{2} v}{\partial x^{2}}+\frac{\partial^{2} v}{\partial y^{2}}+\frac{\partial^{2} v}{\partial \xi^{2}} \geq-2
$$

Now consider the prism

$$
P_{1}=\left\{(x, \xi, y):|x|<\frac{\mathcal{L}}{2},|\xi|<\frac{\mathcal{L}}{2},|y|<\frac{\mathcal{W}}{2}, x-\xi>0\right\}
$$

and the ordinary differential equation problem

$$
h^{\prime \prime}(\tau)=-1, \text { with } h(0)=0 \text { and } h^{\prime}(\mathcal{L})=\epsilon,
$$

where $\epsilon$ is a positive constant. The solution of (3.6) is given by $h(\tau)=-\frac{\tau^{2}}{2}+\tau(\mathcal{L}+\epsilon)$. Define the function $\omega(x, y, \xi) \equiv v(x, y, \xi)-h(x-\xi)$ and take into account (3.5) to get that

$$
\frac{\partial^{2} \omega}{\partial x^{2}}+\frac{\partial^{2} \omega}{\partial y^{2}}+\frac{\partial^{2} \omega}{\partial \xi^{2}} \geq 0
$$

from which, using the strong maximum principle (see Lemma 3.5 in [9]), we conclude that $\omega$ does not achieve its maximum value in $P_{1}$ unless it is a constant function. On the boundary sector defined by $x=\frac{\mathcal{L}}{2},|y| \leq \frac{\mathcal{W}}{2}$, and $-\frac{\mathcal{L}}{2} \leq \xi<\frac{\mathcal{L}}{2}$ we have that

$$
\frac{\partial \omega\left(\frac{\mathcal{L}}{2}, y, \xi\right)}{\partial x}=\frac{\partial u\left(\frac{\mathcal{L}}{2}, y\right)}{\partial x}-h^{\prime}\left(\frac{\mathcal{L}}{2}-\xi\right)=-h^{\prime}\left(\frac{\mathcal{L}}{2}-\xi\right)<0 .
$$

Since $x$ is the outward normal to the domain, $\omega$ does not achieve its maximum on this part of the boundary. On the boundary sector defined by $\xi=-\frac{\mathcal{L}}{2},|y| \leq \frac{\mathcal{W}}{2}$, and $-\frac{\mathcal{L}}{2}<x \leq \frac{\mathcal{L}}{2}$ we have that

$$
\frac{\partial \omega\left(x, y,-\frac{\mathcal{L}}{2}\right)}{\partial \xi}=-\frac{\partial u\left(-\frac{\mathcal{L}}{2}, y\right)}{\partial \xi}+h^{\prime}\left(x+\frac{\mathcal{L}}{2}\right)=h^{\prime}\left(x+\frac{\mathcal{L}}{2}\right)>0,
$$


and since $\xi$ is the inward normal we conclude that the maximum of $\omega$ is not achieved on this part of the boundary either. On the planes $y= \pm \frac{\mathcal{W}}{2},|x|<\frac{\mathcal{L}}{2}$, and $|\xi|<\frac{\mathcal{L}}{2}$ we have that

$$
\frac{\partial \omega\left(x, \pm \frac{\mathcal{W}}{2}, \xi\right)}{\partial y}=\frac{\partial u\left(x, \pm \frac{\mathcal{W}}{2}\right)}{\partial y}-\frac{\partial u\left(\xi, \pm \frac{\mathcal{W}}{2}\right)}{\partial y}=0
$$

and assuming that $\omega$ is not a constant function we have, from Lemma 3.4 in [9], that $\omega$ does not achieve its maximum on these boundary planes either. Therefore, the maximum is achieved at $x=\xi$, and since $\left.\omega(x, y, \xi)\right|_{x=\xi}=0$ we have that the inequality

$$
u(x, y)-u(\xi, y) \leq h(x-\xi)
$$

holds in $\Omega$, which, as can be easily seen, becomes an equality if $\omega$ is a constant function.

Subtracting relation (3.1) from (3.4) and applying the above analysis we obtain that

$$
u(\xi, y)-u(x, y) \leq h(x-\xi),
$$

and hence

$$
|u(x, y)-u(\xi, y)| \leq h(x-\xi) \quad \text { for } x>\xi .
$$

Working in a similar way (or directly obtained by symmetry) for $x<\xi$ we easily see that

$$
|u(x, y)-u(\xi, y)| \leq h(|x-\xi|)-h(0) .
$$

By dividing the last relation by $|x-\xi|$ and taking the limit, we have that $\left|\frac{\partial u}{\partial x}\right| \leq h^{\prime}(0)$ and finally obtain the first of the following inequalities (when $\epsilon \rightarrow 0$ ), while the second can be obtained similarly.

$$
\sup _{(x, y) \in \Omega}\left|\frac{\partial u(x, y)}{\partial x}\right| \leq \mathcal{L}, \quad \sup _{(x, y) \in \Omega}\left|\frac{\partial u(x, y)}{\partial y}\right| \leq \mathcal{W} .
$$

Remark 3.1. As a direct consequence of the above lemma, we easily get the following estimates for the gradient of the solution of the problem (2.1)-(2.2):

$$
-\mathcal{L}+H+\frac{\alpha_{1}+\alpha_{2}}{\mathcal{L}} x+\frac{\alpha_{2}-\alpha_{1}}{2} \leq \frac{\partial \phi}{\partial x} \leq \mathcal{L}+H+\frac{\alpha_{1}+\alpha_{2}}{\mathcal{L}} x+\frac{\alpha_{2}-\alpha_{1}}{2}
$$

and

$$
-\mathcal{W}+\frac{\delta_{1}+\delta_{2}}{\mathcal{W}} y+\frac{\delta_{2}-\delta_{1}}{2} \leq \frac{\partial \phi}{\partial y} \leq \mathcal{W}+\frac{\delta_{1}+\delta_{2}}{\mathcal{W}} y+\frac{\delta_{2}-\delta_{1}}{2}
$$

It is worth noting here that as it follows from (3.8) $\phi_{x}>0$ in the case of large magnetic field $H$. This is consistent with the physical properties of Josephson junctions [2].

Next we obtain sharper estimates by making certain assumptions, on typical junction's size, on the domain. 
LEMma 3.2. (a) Suppose that $\mathcal{I}_{j}$ depends only on the variable $y$. If $\mathcal{L}<2$, then for any classical solution $u(x, y)$ of the problem (2.1)-(2.2) we have

$$
\left|u_{x}\right| \leq \frac{\mathcal{L}^{2} f_{1}}{4-\mathcal{L}^{2}}
$$

where $f_{1}=\max \left|f_{x}(x, y)\right|$.

(b) Suppose that $\mathcal{I}_{j}$ depends only on the variable $x$. If $\mathcal{W}<2$, then for any classical solution $u(x, y)$ of the problem (2.1)-(2.2) we have

$$
\left|u_{y}\right| \leq \frac{\mathcal{W}^{2} f_{2}}{4-\mathcal{W}^{2}}
$$

where $f_{2}=\max \left|f_{y}(x, y)\right|$.

Proof. Arguing in the same manner as in the proof of Lemma 3.1, for $v(x, y, \xi)=$ $u(x, y)-u(\xi, y)$ we obtain

$$
\frac{\partial^{2} v}{\partial x^{2}}+\frac{\partial^{2} v}{\partial y^{2}}+\frac{\partial^{2} v}{\partial \xi^{2}}=\mathcal{I}_{j}(y)(\sin (u(x, y)+f(x, y))-\sin (u(\xi, y)+f(\xi, y)))
$$

and thus (from Lemma 3.1 we already have that $\left|u_{x}\right| \leq \mathcal{L}$ )

$$
\frac{\partial^{2} v}{\partial x^{2}}+\frac{\partial^{2} v}{\partial y^{2}}+\frac{\partial^{2} v}{\partial \xi^{2}} \geq-\mathcal{L}(x-\xi) \quad \text { for } \quad x>\xi
$$

Consider the prism

$$
P_{1}=\left\{(x, \xi, y):|x|<\frac{\mathcal{L}}{2},|\xi|<\frac{\mathcal{L}}{2},|y|<\frac{\mathcal{W}}{2}, x-\xi>0\right\}
$$

and let $h_{1}(\tau)$ be a solution of the problem

$$
h_{1}^{\prime \prime}(\tau)=-\frac{\mathcal{L}+f_{1}}{2} \tau, \quad h_{1}(0)=0, \quad \text { and } \quad h_{1}^{\prime}(\mathcal{L})=\epsilon>0 .
$$

Obviously for $h_{1}=h_{1}(x-\xi)$ we have

$$
\frac{\partial^{2} h_{1}}{\partial x^{2}}+\frac{\partial^{2} h_{1}}{\partial y^{2}}+\frac{\partial^{2} h_{1}}{\partial \xi^{2}}=-\left(\mathcal{L}+f_{1}\right)(x-\xi) .
$$

Subtracting (3.15) from (3.13) for $\omega(x, y, \xi) \equiv v(x, y, \xi)-h_{1}(x-\xi)$ we obtain that

$$
\frac{\partial^{2} \omega}{\partial x^{2}}+\frac{\partial^{2} \omega}{\partial y^{2}}+\frac{\partial^{2} \omega}{\partial \xi^{2}} \geq 0
$$

Arguing analogously to the proof of Lemma 3.1 we have

$$
\left|u_{x}(x, y)\right| \leq h_{1}^{\prime}(0)=\frac{\mathcal{L}+f_{1}}{4} \mathcal{L}^{2}+\epsilon,
$$

and passing to the limit when $\epsilon \rightarrow 0$,

$$
\left|u_{x}(x, y)\right| \leq \frac{\mathcal{L}+f_{1}}{4} \mathcal{L}^{2} \equiv \mathcal{L}_{1} .
$$


Returning back to (3.12) and taking into account that now $\left|u_{x}\right| \leq \mathcal{L}_{1}$ we obtain

$$
\frac{\partial^{2} v}{\partial x^{2}}+\frac{\partial^{2} v}{\partial y^{2}}+\frac{\partial^{2} v}{\partial \xi^{2}} \geq-\left(\mathcal{L}_{1}+f_{1}\right)(x-\xi) \quad \text { for } \quad x>\xi .
$$

Construct the function

$$
h_{2}^{\prime \prime}(\tau)=-\frac{\mathcal{L}_{1}+f_{1}}{2} \tau, \quad h_{2}(0)=0, \quad \text { and } \quad h_{2}^{\prime}(\mathcal{L})=\epsilon .
$$

In a similar manner as above we conclude that

$$
\left|u_{x}(x, y)\right| \leq h_{2}^{\prime}(0)=\frac{\mathcal{L}_{1}+f_{1}}{4} \mathcal{L}^{2}+\epsilon
$$

and letting $\epsilon \rightarrow 0$ we have

$$
\left|u_{x}(x, y)\right| \leq \frac{\mathcal{L}_{1}+f_{1}}{4} \mathcal{L}^{2}=\left(\frac{\mathcal{L}}{2}\right)^{4}\left(\mathcal{L}+f_{1}\right)+\left(\frac{\mathcal{L}}{2}\right)^{2} f_{1} \equiv \mathcal{L}_{2} .
$$

Continuing this procedure we obtain the sequence of the bounds for $\left|u_{x}\right|$,

$$
\mathcal{L}_{n}=\left(\frac{\mathcal{L}}{2}\right)^{2 n} \mathcal{L}+f_{1}\left[\left(\frac{\mathcal{L}}{2}\right)^{2 n}+\left(\frac{\mathcal{L}}{2}\right)^{2(n-1)}+\cdots+\left(\frac{\mathcal{L}}{2}\right)^{4}+\left(\frac{\mathcal{L}}{2}\right)^{2}\right] .
$$

If $\mathcal{L}<2$, then $\left(\frac{\mathcal{L}}{2}\right)^{2 n} \mathcal{L} \rightarrow 0$ when $n \rightarrow \infty$, and the second term

$$
f_{1}\left[\left(\frac{\mathcal{L}}{2}\right)^{2 n}+\cdots+\left(\frac{\mathcal{L}}{2}\right)^{2}\right] \rightarrow f_{1} \frac{\mathcal{L}^{2}}{4-\mathcal{L}^{2}}
$$

This concludes the proof of part (a); the proof of part (b) is similar.

Remark 3.2. Recall that $u \equiv \phi-f$ and use the above lemma to obtain the following estimates in terms of $\phi$ :

$$
\text { 9) }-\frac{\mathcal{L}^{2} f_{1}}{4-\mathcal{L}^{2}}+H+\frac{\alpha_{1}+\alpha_{2}}{\mathcal{L}} x+\frac{\alpha_{2}-\alpha_{1}}{2} \leq \frac{\partial \phi}{\partial x} \leq \frac{\mathcal{L}^{2} f_{1}}{4-\mathcal{L}^{2}}+H+\frac{\alpha_{1}+\alpha_{2}}{\mathcal{L}} x+\frac{\alpha_{2}-\alpha_{1}}{2} \text {. }
$$

In particular, if $f_{1}=H=\alpha_{1}=\alpha_{2} \equiv 0$, then $\frac{\partial \phi}{\partial x} \equiv 0$.

$$
\text { 20) }-\frac{\mathcal{W}^{2} f_{2}}{4-\mathcal{W}^{2}}+\frac{\delta_{1}+\delta_{2}}{\mathcal{W}} y+\frac{\delta_{2}-\delta_{1}}{2} \leq \frac{\partial \phi}{\partial y} \leq \frac{\mathcal{W}^{2} f_{2}}{4-\mathcal{W}^{2}}+\frac{\delta_{1}+\delta_{2}}{\mathcal{W}} y+\frac{\delta_{2}-\delta_{1}}{2}
$$

In particular, if $f_{2}=\delta_{1}=\delta_{2} \equiv 0$, then $\frac{\partial \phi}{\partial y} \equiv 0$.

Remark 3.3. If $\delta_{1}=\delta_{2}=0, \mathcal{I}_{j}=\mathcal{I}_{j}(x)$, and $\mathcal{W}<2$, then our problem becomes one-dimensional: $\phi(x, y)=\phi(x)$ and (2.1)-(2.2) take the form

$$
\phi^{\prime \prime}(x)=\mathcal{I}_{j}(x) \sin \phi(x), \quad \phi^{\prime}\left(-\frac{\mathcal{L}}{2}\right)=H-\alpha_{1}, \quad \phi^{\prime}\left(\frac{\mathcal{L}}{2}\right)=H+\alpha_{2} .
$$

Lemma 3.3. The first order derivatives of the classical solution of problem (2.1)(2.2) are Hölder continuous with the Hölder coefficient and exponent depending only on $\left\|\frac{\partial \phi}{\partial x}\right\|_{L^{2}(\Omega)},\left\|\frac{\partial \phi}{\partial y}\right\|_{L^{2}(\Omega)}$, and $\alpha_{1}, \alpha_{2}, \delta_{1}, \delta_{2}, \mathcal{L}$, and $\mathcal{W}$.

The bounds on the Hölder norm of the gradient follow from Theorem 9.11 in [9]. 
4. Existence and uniqueness. In this section we show, under certain conditions, the existence and the uniqueness of a solution of the PDE problem (3.1)-(3.2) (and therefore of the problem (2.1)-(2.2)). We start by giving the definition of the generalized solution.

Definition 4.1. We call a function $u(x, y) \in C^{1, \alpha}(\bar{\Omega}) \cap W_{2}^{2}(\Omega)$ a generalized solution of the PDE problem (3.1)-(3.2) if it satisfies the integral identity

$$
\int_{\Omega}\left(u_{x x}+u_{y y}-\mathcal{I}_{j} \sin (u+f)-g\right) \psi d x d y=0 \quad \forall \psi \in L^{2}(\Omega)
$$

and the boundary conditions (3.2), where $g \equiv-\frac{\alpha_{1}+\alpha_{2}}{\mathcal{L}}-\frac{\delta_{1}+\delta_{2}}{\mathcal{W}}$.

4.1. Existence. We start by assuming that $\mathcal{I}_{j}$ is smooth and consider the auxiliary problem

$$
\begin{gathered}
\Delta v=\kappa \mathcal{I}_{j}\left(\sin (v+f)-\frac{1}{\mu\left(\Omega_{j}\right)} \int_{\Omega_{j}} \sin (v+f) d x d y\right) \text { in } \Omega \\
\frac{\partial v}{\partial n}=0 \quad \text { on } \partial \Omega
\end{gathered}
$$

and

$$
\frac{1}{\mu(\Omega)} \int_{\Omega} v d x d y=\zeta
$$

where $\kappa \in[0,1]$ and $\zeta$ is an arbitrary fixed real number. Recall that by $\mu(\Omega)$ we denote the measure of $\Omega$. We will show that a solution $v \in C^{1, \gamma}(\bar{\Omega}) \cap C^{3}(\Omega)$ of the auxiliary problem (4.2)-(4.4) exists. For this we define $\psi \equiv v-\zeta$ and write the above problem in the following equivalent form:

$$
\begin{gathered}
\Delta \psi=\kappa \mathcal{I}_{j}\left[\sin (\psi+\zeta+f)-\frac{1}{\mu\left(\Omega_{j}\right)} \int_{\Omega_{j}} \sin (\psi+\zeta+f) d x d y\right] \text { in } \Omega, \\
\frac{\partial \psi}{\partial n}=0 \quad \text { on } \partial \Omega,
\end{gathered}
$$

and

$$
\int_{\Omega} \psi d x d y=0
$$

As we easily see, the only difference between (3.1) and (4.5) is a bounded constant term on the right-hand side. Hence the estimates obtained in the lemmas in the previous section hold for (4.5)-(4.6) too. We can also observe that $\psi$ becomes zero at least at one point in $\Omega$ so that using the estimate of the gradient (which are independent of the $\max |\psi|$ ) we can obtain a bound for the maximum of $|\psi|$ in the domain $\bar{\Omega}$. We are in the position now to use the Leray-Schauder theorem [9, Theorem 11.3] (see the appendix) to prove the existence of the generalized solution of problem (4.2)-(4.4).

LERAY-SCHAUDER THEOREM. Let $T$ be a compact mapping from a Banach space $\mathcal{B}$ to itself, and suppose there exists a constant $M$ such that $\|u\|_{\mathcal{B}}<M$ for all $u \in \mathcal{B}$ and $\kappa \in[0,1]$ satisfying $u=\kappa T u$. Then $T$ has a fixed point. 
We now address the case of a nonsmooth function $\mathcal{I}_{j}$.

LEMma 4.2. For the classical solution of the PDE problem (4.2)-(4.4) for $\zeta=1$ the following inequality holds:

$$
\int_{\Omega}\left[\left(\frac{\partial^{2} v}{\partial x^{2}}\right)^{2}+2\left(\frac{\partial^{2} v}{\partial x \partial y}\right)^{2}+\left(\frac{\partial^{2} v}{\partial y^{2}}\right)^{2}\right] d x d y \leq 4 \mu\left(\Omega_{j}\right) .
$$

Proof. We square both sides of (4.2), integrate them two times (using integration by parts for the second term on the left-hand side). Then the use of the boundary conditions easily gives the above bound.

Obviously the classical solution $v$ satisfies the integral identity (4.1), i.e.,

$\int_{\Omega}\left(\frac{\partial^{2} v}{\partial x^{2}}+\frac{\partial^{2} v}{\partial y^{2}}-\mathcal{I}_{j}^{\delta} \sin (v+f)+\frac{1}{\mu(\Omega)} \int_{\Omega_{j}} \sin (v+f) d x d y\right) \psi d x d y=0 \forall \psi \in L^{2}(\Omega)$.

Taking the limit, as $\delta \rightarrow 0$ we readily obtain the existence of the generalized solution.

Let us fix arbitrarily $\zeta=\zeta_{0}$. For this $\zeta_{0}$ we find the generalized solution of the problem (4.2)-(4.4) for $\kappa=1$. In order to obtain the existence of the original problem we need to find boundary conditions such that

$$
R(c)=c,
$$

where

$$
c \equiv \mu\left(\Omega_{j}\right)\left(\frac{\alpha_{1}+\alpha_{2}}{\mathcal{L}}+\frac{\delta_{1}+\delta_{2}}{\mathcal{W}}\right)
$$

and

$$
R(c) \equiv \int_{\Omega_{j}} \sin (v+f) d x d y
$$

Note that $R$ satisfies the inequality $|R|<\mu\left(\Omega_{j}\right)$, and observe, assuming that $R$ continuously depends on $c$, that it is impossible to have $R<c$ for $c$ varying from $\mu\left(\Omega_{j}\right)$ to $-\mu\left(\Omega_{j}\right)$. Hence there exists such $c_{0}$ that verifies (4.10). For this $c_{0}$ the solution of the auxiliary problem (4.2)-(4.4) coincides with the solution of the original one (2.1)-(2.2). Mark that the assumption on the continuity of $R$ is satisfied in the cases of the uniqueness of the solution of the auxiliary problem. Such uniqueness can be proved following an analysis similar to the one presented in Theorem 4.4. From the above we readily obtain the following theorem.

THEOREM 4.3. If the solution of the problem (4.2)-(4.4) is unique, then for any $\zeta \in \mathbb{R}$ we can find values for $H, \alpha_{1}, \alpha_{2}, \delta_{1}$, and $\delta_{2}$ for which there exists a generalized solution $\phi$ of the problem (2.1)-(2.2) such that

$$
\frac{1}{\mu(\Omega)} \int_{\Omega}(\phi-f) d x d y=\zeta .
$$

Let us note that Theorem 4.3 also holds if condition (4.11) is replaced by

$$
\left.(\phi-f)\right|_{\left(x_{0}, y_{0}\right)}=\zeta, \quad\left(x_{0}, y_{0}\right) \in \Omega .
$$

To prove this, one has to carry out an analysis similar to the above, which is lengthy and tedious and so will not be presented here. 
4.2. Uniqueness. It has been observed both numerically and experimentally $[2,3,4]$ and it is intuitively expected that our PDE problem might have more than one nontrivial solution. In the case when $\mathcal{L}<2$ and $\mathcal{W}<2$ we can easily see from Lemma 3.2 that the only solution is $n \pi, n=0, \pm 1, \pm 2, \ldots$. An extensive theoretical and experimental bifurcation analysis is under way and will be presented elsewhere. Nevertheless, as we show below, under certain conditions only one solution exists.

THEOREM 4.4. Assume that either

(a) $\mathcal{L}<2, w<\sqrt{2}, \mathcal{I}_{j}=\mathcal{I}_{j}(y)$, and $H=\alpha_{1}=\alpha_{2}=0$, or

(b) $\mathcal{W}<2, \ell<\sqrt{2}, \mathcal{I}_{j}=\mathcal{I}_{j}(x)$, and $\delta_{1}=\delta_{2}=0$.

Then the generalized solution $u$ of (3.1)-(3.2) satisfying the condition

$$
\frac{1}{\mu(\Omega)} \int_{\Omega} u d x d y=\zeta
$$

where $\zeta$ is an arbitrarily given constant, is unique.

Proof. From Lemma 3.2 it follows that $u_{x} \equiv 0$. Thus we have

$$
\begin{gathered}
u_{y y}=\mathcal{I}_{j}(y) \sin (u+f)-\frac{\delta_{1}+\delta_{2}}{\mathcal{W}} \text { in } \Omega, \\
u_{y}\left( \pm \frac{\mathcal{W}}{2}\right)=0
\end{gathered}
$$

and

$$
\int_{-\frac{\mathcal{W}}{2}}^{\frac{\mathcal{W}}{2}} u d y=\zeta
$$

Suppose now that there exist two different solutions $u$ and $v$, both satisfying condition (4.15), i.e.,

$$
\int_{-\frac{\mathcal{W}}{2}}^{\frac{\mathcal{W}}{2}} u d y=\int_{-\frac{\mathcal{W}}{2}}^{\frac{\mathcal{W}}{2}} v d y .
$$

This implies that $u$ and $v$ cross each other. Now let $\sigma \equiv u-v$ and observe that

$$
\sigma_{y y}=\mathcal{I}_{j}(y)(\sin (u(y)+f(y))-\sin (v(y)+f(y)))=\mathcal{I}_{j}(y) \sigma \cos \theta .
$$

Suppose that $u$ and $v$ intersect at a point $y_{0}$. Consider the two cases

$$
\text { ( } \alpha) y_{0} \notin\left[-\frac{w}{2}, \frac{w}{2}\right], \quad(\beta) y_{0} \in\left[-\frac{w}{2}, \frac{w}{2}\right] .
$$

In $(\alpha)$ consider the case $y_{0} \in\left(-\frac{\mathcal{W}}{2}, \frac{w}{2}\right]$. In the interval $\left(-\frac{\mathcal{W}}{2}, y_{0}\right)$ we have $\sigma_{y y}=0$ and $\sigma_{y}\left(-\frac{\mathcal{W}}{2}\right)=\sigma\left(y_{0}\right)=0$. Hence $\sigma \equiv 0 \in\left(-\frac{\mathcal{W}}{2}, y_{0}\right)$. Therefore, due to the analyticity of $\sigma$ in $\left(-\frac{\mathcal{W}}{2}, \frac{w}{2}\right]$ we have $\sigma \equiv 0 \in\left(-\frac{\mathcal{W}}{2}, \frac{w}{2}\right]$. Similarly we can consider the case $y_{0} \in\left[\frac{w}{2}, \frac{\mathcal{W}}{2}\right)$.

Consider now the $(\beta)$ case. Multiplying (4.17) by $\sigma$ and integrating by parts we get

$$
\int_{y_{0}}^{\frac{\mathcal{W}}{2}} \sigma_{y}^{2} d y \leq \int_{y_{0}}^{\frac{\mathcal{W}}{2}} \mathcal{I}_{j} \sigma^{2} d y=\int_{y_{0}}^{\frac{w}{2}} \sigma^{2} d y .
$$


Applying the Poincaré inequality we obtain

$$
\int_{y_{0}}^{\frac{w}{2}} \sigma_{y}^{2} d y \leq \int_{y_{0}}^{\frac{\mathcal{W}}{2}} \sigma_{y}^{2} d y \leq \int_{y_{0}}^{\frac{w}{2}} \sigma^{2} d y \leq \frac{w^{2}}{2} \int_{y_{0}}^{\frac{w}{2}} \sigma_{y}^{2} d y .
$$

Due to the assumption $w<\sqrt{2}$ we have $\int_{y_{0}}^{\frac{w}{2}} \sigma_{y}^{2} d y=0$, and therefore $\sigma_{y} \equiv 0$. Since $\sigma\left(y_{0}\right)=0$ we have $\sigma \equiv 0$ and $u \equiv v$.

We note that there are other cases where one might be able to show this uniqueness. For example, we have shown that if we assume that the window is such that $\ell<\sqrt{2}$ and $w<\sqrt{2}$, then the generalized solution $u$ of (3.1)-(3.2) satisfying the condition

$$
\frac{1}{\mu(\Omega)} \int_{\Omega} u d x d y=\zeta
$$

where $\zeta$ is an arbitrarily given constant, is unique. The proof of this statement is similar to the proof of the previous theorem. Since it is rather technical, tedious, and lengthy it is not included here.

In a manner similar to the above theorem it can be shown that there exist cases where the solution of the auxiliary problem is unique.

5. Additional estimates. In this section we obtain estimates of the gradient of the solution of the problem in some special cases that are of physical interest. As discussed in section 2 it is useful $[3,4,5]$ to characterize the solutions of $(2.1)-(2.2)$ by their oscillations, defined by (2.4). In what follows we derive estimates of the gradient of the solution as a function of its oscillations in the $x$ - and $y$-directions.

Lemma 5.1. For any classical solution $\phi(x, y)$ of the problem $(2.1)-(2.2)$ we have that

$$
\left|\frac{\partial \phi}{\partial x}\right| \leq \sqrt{2 o s c_{x} \phi+\left(H+\alpha_{2}\right)^{2}}
$$

and

$$
\left|\frac{\partial \phi}{\partial y}\right| \leq \sqrt{2 o s c_{y} \phi+\delta_{2}^{2}}
$$

Proof. We follow the analysis of Lemma 3.1, with the main difference being in the construction of the barrier $h$. Specifically we set $v(x, y, \xi) \equiv \phi(x, y)-\phi(\xi, y)$ and define $h(\tau)$ as the solution of the problem

$$
h^{\prime \prime}(\tau)=-1, \quad h(0)=0, \quad h\left(\tau^{*}\right)=o s c_{x} \phi,
$$

where $\tau^{*}$ will be defined later. We need to compare the functions $v$ and $h(x-\xi)$ in the prism $P_{2} \cap\{x-\xi<\mathcal{L}\}$, where

$$
P_{2}=\left\{(x, \xi, y):|x|<\frac{\mathcal{L}}{2},|\xi|<\frac{\mathcal{L}}{2},|y|<\frac{\mathcal{W}}{2}, \tau^{*}>x-\xi>0\right\},
$$

whose cross-section along the $y$-axis is given in Figure 5.1. Obviously (see (3.7)) we have $\Delta(v-h) \geq 0$ in $P_{2} \cap\{x-\xi<\mathcal{L}\}$, and hence the maximum is not achieved in the interior of $P_{2} \cap\{x-\xi<\mathcal{L}\}$. We need to check the boundary. When $x=\xi$ and $y \in\left[-\frac{\mathcal{W}}{2}, \frac{\mathcal{W}}{2}\right]$ we have $v-h=0$. 


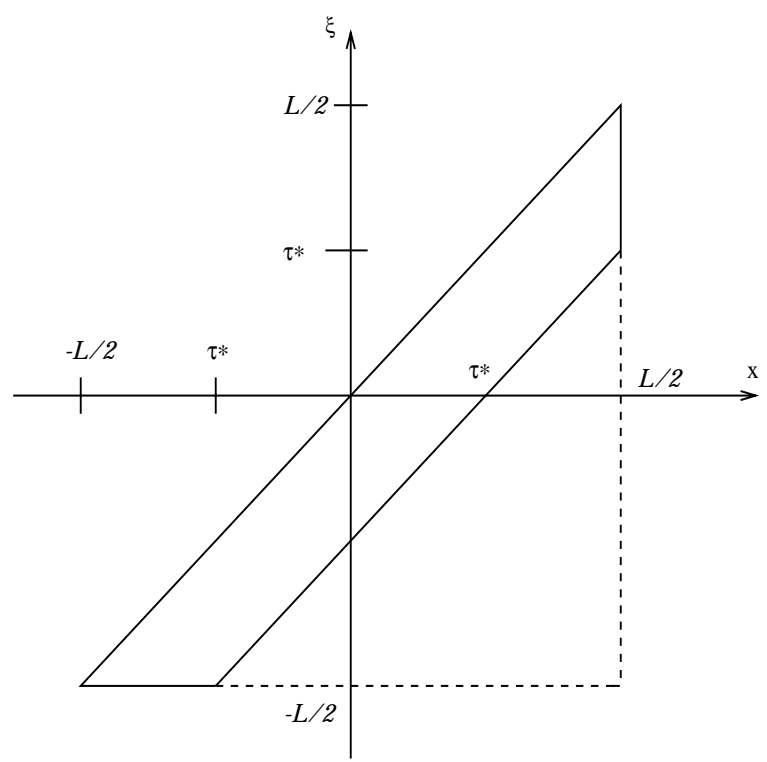

FIG. 5.1. A cross-section of domain $P_{2}$ along a plane in the $y$-direction.

For $x-\xi=\tau^{*}$ we obtain $v-o s c_{x} \phi \leq 0$ for $y \in\left[-\frac{\mathcal{W}}{2}, \frac{\mathcal{W}}{2}\right]$. For $x=\frac{\mathcal{L}}{2}, \xi \in$ $\left(-\tau^{*}+\frac{\mathcal{L}}{2}, \frac{\mathcal{L}}{2}\right)$, and $y \in\left[-\frac{\mathcal{W}}{2}, \frac{\mathcal{W}}{2}\right]$ we have that

$$
\left.\frac{\partial(v-h)}{\partial x}\right|_{x=\frac{\mathcal{L}}{2}}=H+\alpha_{2}-h^{\prime}\left(\frac{\mathcal{L}}{2}-\xi\right) .
$$

Similarly if $\xi=-\frac{\mathcal{L}}{2}, x \in\left(-\frac{\mathcal{L}}{2},-\frac{\mathcal{L}}{2}+\tau^{*}\right)$, and $y \in\left[-\frac{\mathcal{W}}{2}, \frac{\mathcal{W}}{2}\right]$, then

$$
\left.\frac{\partial(v-h)}{\partial \xi}\right|_{\xi=-\frac{\mathcal{L}}{2}}=-\left(H-\alpha_{1}\right)+h^{\prime}\left(x+\frac{\mathcal{L}}{2}\right) .
$$

Therefore if $h^{\prime}>H+\alpha_{2}$ (note that $H, \alpha_{1}, \alpha_{2}, \delta_{1}, \delta_{2}$ are positive constants), then

$$
\left.\frac{\partial(v-h)}{\partial x}\right|_{x=\frac{\mathcal{L}}{2}}<0,\left.\quad \frac{\partial(v-h)}{\partial \xi}\right|_{\xi=-\frac{\mathcal{L}}{2}}>0
$$

and hence we do not have a maximum on these parts of the boundary of $P_{2}$. Furthermore, since for $y= \pm \frac{\mathcal{L}}{2}, x \in\left(-\frac{\mathcal{L}}{2}, \frac{\mathcal{L}}{2}\right)$, and for $\xi \in\left(-\frac{\mathcal{L}}{2}, \frac{\mathcal{L}}{2}\right)$ and $\xi \in\left(-\frac{\mathcal{L}}{2}, \frac{\mathcal{L}}{2}\right)$ and $0<x-\xi<\tau^{*}$ we have $\frac{\partial(v-h)}{\partial y}=0$, we conclude (see Lemma 3.4 in [8]) that we do not have a maximum here either. It remains to choose $\tau^{*}$ such that $h^{\prime}(\tau)>H+\alpha_{2}$ for $\tau \in\left[0, \tau^{*}\right]$. For this we get

$$
\tau^{*}<-\left(H+\alpha_{2}\right)+\sqrt{\left(H+\alpha_{2}\right)^{2}+2 o s c_{x} \phi} .
$$

As previously we have that

$$
\left|\phi_{x}(x, y)\right| \leq h^{\prime}(0)=\frac{o s c_{x} \phi}{\tau^{*}}+\frac{\tau^{*}}{2} .
$$

It can be seen that the minimum of $h^{\prime}(0)$ with respect to $\tau^{*}$ is achieved when $\tau^{*}=$ $-\left(H+\alpha_{2}\right)+\sqrt{\left(H+\alpha_{2}\right)^{2}+2 o s c_{x} \phi}$, from which we obtain relation (5.1). 
For $\tau^{*} \geq \mathcal{L}$ the only difference is the absence of the boundary $x-\xi=\tau^{*}$, and the boundaries $x=\frac{\mathcal{L}}{2}, \xi \in\left(-\tau^{*}+\frac{\mathcal{L}}{2}\right), \frac{\mathcal{L}}{2}, y \in\left[-\frac{\mathcal{W}}{2}, \frac{\mathcal{W}}{2}\right]$ become $x=\frac{\mathcal{L}}{2}, \xi \in\left(-\frac{\mathcal{L}}{2}, \frac{\mathcal{L}}{2}\right.$, $y \in\left[-\frac{\mathcal{W}}{2}, \frac{\mathcal{W}}{2}\right]$, and $\xi=-\frac{\mathcal{L}}{2}, x \in\left(-\frac{\mathcal{L}}{2},-\frac{\mathcal{L}}{2}+\tau^{*}\right), y \in\left[-\frac{\mathcal{W}}{2}, \frac{\mathcal{W}}{2}\right]$ become $\xi=-\frac{\mathcal{L}}{2}$, $x \in\left(-\frac{\mathcal{L}}{2}, \frac{\mathcal{L}}{2}\right), y \in\left[-\frac{\mathcal{W}}{2}, \frac{\mathcal{W}}{2}\right]$. We work similarly for the case $\tau^{*}<\mathcal{L}$

One can obtain relation (5.2) working similarly for the $y$ variable.

Remark. We conclude the discussion in this section by observing that in the case where $\alpha_{1}=\alpha_{2}=\alpha$ and $\delta_{1}=\delta_{2}=\delta$ the numerical experiments that we have conducted, shown in section 7 , indicate that a solution of the PDE problem (2.1)(2.2) is symmetric along the axis $y=0$. To obtain this solution one can reduce the PDE problem $(2.1)$ to the domain defined by $\left(-\frac{\mathcal{L}}{2}, \frac{\mathcal{L}}{2}\right) \times\left(-\frac{\mathcal{W}}{2}, 0\right)$ together with the boundary conditions $\frac{\partial \phi(x, y)}{\partial x}=H \pm \alpha$ on $x= \pm \mathcal{L} / 2, \frac{\partial \phi(x, y)}{\partial y}=\delta$ on $y=-\mathcal{W} / 2$, and $\frac{\partial \phi(x, y)}{\partial y}=0$ on $y=0$. The solution to the original problem is obtained by applying symmetry across the $y$-axis. For the reduced problem the estimates (3.9) and (4.8) obtained above can be improved to become

$$
-\frac{\mathcal{W}}{2}-\frac{2 \delta}{\mathcal{W}} y \leq \frac{\partial \phi(x, y)}{\partial y} \leq \frac{\mathcal{W}}{2}-\frac{2 \delta}{\mathcal{W}} y
$$

and

$$
\begin{aligned}
& \int_{-\frac{\mathcal{L}}{2}}^{\frac{\mathcal{L}}{2}} \int_{-\frac{\mathcal{W}}{2}}^{0}\left[\left(\frac{\partial^{2} \phi}{\partial x^{2}}\right)^{2}+2\left(\frac{\partial^{2} \phi}{\partial x \partial y}\right)^{2}+\left(\frac{\partial^{2} \phi}{\partial y^{2}}\right)^{2}\right] d x d y \\
& \leq \frac{1}{2} \mu\left(\Omega_{j}\right)\left[1+4\left(\frac{\alpha}{\mathcal{L}}+\frac{\delta}{\mathcal{W}}\right)\right]+2 \alpha \frac{H \mathcal{W}}{\mathcal{L}}+5 \delta^{2} \frac{\mathcal{L}}{\mathcal{W}}+2 \alpha \delta
\end{aligned}
$$

respectively.

In the particular case when $H=\delta=0$ and the junction is placed symmetrically inside $\Omega$, we have observed the existence of a solution for which the phase is equal to a constant along the line $x=0$ (see the top of Figure 7.2). Although we are unable to prove their existence, such solutions have been observed in practice, and their physical justification is well established in the case where no extra fluxons have entered the interior of the window $[4,5]$. For this type of solution we are able to obtain the following estimations of its size.

LEMma 5.2. For any classical solution $\phi(x, y)$ of the problem $(2.1)-(2.2)$ with $H, \delta=0$ and $\alpha_{i}=a$, for which $\phi=k, k$ is a constant, at $x=0$, we have that

$$
\begin{aligned}
& k+\frac{x^{2}}{2}+\left(\frac{\mathcal{L}}{2}-\alpha\right) x \leq \phi \leq k-\frac{x^{2}}{2}-\left(\alpha+\frac{\mathcal{L}}{2}\right) x \quad \text { for } \quad-\frac{\mathcal{L}}{2} \leq x \leq 0, \\
& k+\frac{x^{2}}{2}-\left(\frac{\mathcal{L}}{2}-\alpha\right) x \leq \phi \leq k-\frac{x^{2}}{2}+\left(\frac{\mathcal{L}}{2}+\alpha\right) x \quad \text { for } \quad 0 \leq x \leq \frac{\mathcal{L}}{2} .
\end{aligned}
$$

Proof. To obtain relation (5.5) we consider the domain $\Omega_{1} \equiv\left(-\frac{\mathcal{L}}{2}, 0\right) \times\left(-\frac{\mathcal{W}}{2}, \frac{\mathcal{W}}{2}\right)$, and from (3.1) we have

$$
-\beta-1 \leq \frac{\partial^{2} u(x, y)}{\partial x^{2}}+\frac{\partial^{2} u(x, y)}{\partial y^{2}} \leq-\beta+1,
$$

where $\beta=\frac{2 \alpha}{\mathcal{L}}$. We define now the function $g(x) \equiv \frac{1-\beta}{2}\left(x+\frac{\mathcal{L}}{2}\right)^{2}+\epsilon x$, where $\epsilon>0$ and $v \equiv u-g$. Obviously we have that

$$
\frac{\partial^{2} v(x, y)}{\partial x^{2}}+\frac{\partial^{2} v(x, y)}{\partial y^{2}} \leq 0
$$


thus $v$ does not achieve its minimum in $\Omega_{1}$ (unless it is a constant). It does not achieve it on the boundary lines $x=-\frac{\mathcal{L}}{2}, y \in\left[-\frac{\mathcal{W}}{2}, \frac{\mathcal{W}}{2}\right]$ (since the derivative in the $x$-direction is negative) and $y= \pm \frac{\mathcal{W}}{2}$ for $x \in\left(-\frac{\mathcal{L}}{2}, 0\right)$ (see Lemma 3.4, p. 34 in [9]) either. So, we conclude that the minimum of $v$ occurs at $x=0$, and we have that

$$
v \geq\left.\min (u-g)\right|_{x=0}=k-\frac{\mathcal{L}^{2}}{8},
$$

and therefore

$$
u \geq k+\frac{1-\beta}{2} x^{2}+(1-\beta) \frac{\mathcal{L}}{2} x-\frac{\beta}{8} \mathcal{L}^{2}+\epsilon x .
$$

We now set $\hat{v} \equiv u-\hat{g}$, where $\hat{g}(x) \equiv-\frac{\beta+1}{2}\left(x+\frac{\mathcal{L}}{2}\right)^{2}-\epsilon x$, where $\epsilon>0$, from which we have that

$$
\frac{\partial^{2} \hat{v}(x, y)}{\partial x^{2}}+\frac{\partial^{2} \hat{v}(x, y)}{\partial y^{2}} \geq 0
$$

Using arguments similar to those above we can show that $\hat{v} \leq\left.\max \hat{v}\right|_{x=0}$ to obtain

$$
u \leq k-\frac{\beta+1}{2} x^{2}-(\beta+1) \frac{\mathcal{L}}{2} x-\frac{\beta}{8} \mathcal{L}^{2}-\epsilon x .
$$

To conclude the proof of relation (5.5) we simply repeat the above analysis for the domain $\left(0, \frac{\mathcal{L}}{2}\right) \times\left(-\frac{\mathcal{W}}{2}, \frac{\mathcal{W}}{2}\right)$, use the fact that $\epsilon$ is an arbitrary positive constant, and simply go from the function $u$ to the function $\phi$.

6. Linearization. For the numerical solution of the semilinear elliptic PDE problem (2.1)-(2.2) one can linearize the PDE equation by means of the following fixed point iteration scheme:

$$
L \phi^{(i)} \equiv \Delta \phi^{(i)}-\mathcal{I}_{j} r \phi^{(i)}=\mathcal{I}_{j}\left(\sin \left(\phi^{(i-1)}\right)-r \phi^{(i-1)}\right), \quad i=1,2, \ldots,
$$

where $r \equiv r(x, y)$ is a relaxation function to accelerate the convergence, and it can be any nonzero function. We start these iterations using an initial guess $u^{(0)}$ of the solution $u$ obtained using one of the approaches described in [4], and we terminate them when the max-norm of the difference of two successive approximations of the solution vector $\left(\left\|\phi^{(i)}-\phi^{(i-1)}\right\|_{\infty}\right)$ or the max-norm of the residual of the problem $\left(\left\|\Delta \phi^{(i)}-\mathcal{I} \sin \phi^{(i)}\right\|_{\infty}\right)$ is less than a given tolerance. Two obvious choices for that parameter are $r(x, y)=c$ (constant function) and $r(x, y)=\cos \left(\phi^{(i-1)}(x, y)\right)$. With the latter one, the iteration scheme (6.1) reduces to the well-known Newton iterative method [14]. The implementation and the performance of this quadratically converging method is given in [4], and its convergence analysis is under way and will be presented elsewhere. For the convergence of (6.1) when $r$ is a positive constant we have the following theorem.

THEOREM 6.1. If $c \equiv c(r)$ is the measure of the smallest eigenvalue of the operator $L$, then the iterative method (6.1) converges, from any initial guess $\phi^{(0)}$, to the solution of (3.1)-(3.2) if

$$
\frac{1}{c}\left(\frac{1}{2}+r\right)<1
$$


Proof. If we denote by $e^{(i)} \equiv \phi-\phi^{(i)}$ the error at the $i$ th iteration, we see that for $i=1,2, \ldots$ we have

$$
L e^{(i)}=\mathcal{I}_{j}\left[\cos \left(\frac{e^{(i-1)}}{2}+\phi\right) \sin \left(\frac{e^{(i-1)}}{2}\right)-r e^{(i-1)}\right],
$$

from which we obtain

$$
\left\|L e^{(i)}\right\| \leq\left\|\cos \left(\frac{e^{(i-1)}}{2}+\phi\right) \sin \left(\frac{e^{(i-1)}}{2}\right)\right\|+r\left\|e^{(i-1)}\right\| .
$$

By expanding the sine term and dropping the cosine term in the above relation we have for $i=1,2, \ldots$ that

$$
\left\|L e^{(i)}\right\| \leq\left(\frac{1}{2}+r\right)\left\|e^{(i-1)}\right\|
$$

from which relation (6.2) can be easily obtained by requiring the amplification factor to be less than 1.

It is worth pointing out here that the lack of convergence, as one increases the current, of the Newton iterative scheme defined above reflects the dynamical instability of the static solution in the time-dependent sine-Gordon system.

7. Numerical experiments and physical relevance. Using the proposed PDE model we have built a powerful simulation tool that accurately and effectively models window Josephson junctions. Our implementation, described in detail in [4], is based on the ELLPACK infrastructure [16], and its basic components are as follows: a uniform discretization of the domain $\Omega$ using a tensor product of $n \times n$ grid lines, the Newton linearization scheme, and the discretization of the PDE problem (3.1)(3.2) using the standard 5-point-star finite difference method. For all experiments we have used a junction $\Omega=[0,12] \times[0,3]$, and unless otherwise stated the window $\Omega_{j}$ has sizes $\ell=10$ and $w=1$ in the $x$ - and $y$-direction, respectively, and is placed in the center of $\Omega$. The boundary conditions were selected such that $\alpha_{1}=\alpha_{2} \equiv \alpha$ and $\delta_{1}=\delta_{2} \equiv \delta$.

In Figure 7.1 we present the properties of the Newton linearization scheme with which we obtained all the numerical data reported here. On the left we see the history of convergence during the first four iterations. Specifically we plot, in $\log -\log$ scale, the quantity $\left\|\phi^{(i)}-\phi^{(i-1)}\right\|_{\infty}$ versus the iteration number $i$ for $i=1,2,3,4$ with $n=20,40$, and 60 , and we easily see the quadratic rate of convergence. To measure the accuracy obtained in the fourth iteration, we plot in the middle panel the infinity and the $L_{2}$ norms of the residual $\left(\Delta \phi-\mathcal{I}_{j} \sin \phi\right)$ versus $n$ in semilog scale. The theoretically expected [16] second order convergence, with respect to discretization stepsize, of the 5-point-star discretization scheme used to solve the linear problems at every step in (6.1) can be easily verified. The time complexity of the Newton iterative algorithm is presented in the right panel, where we plot the per-iteration CPU time required versus $n$. As is easily seen this is approximately $n^{3}$.

To understand the structure of the solutions for various boundary conditions and confirm the obtained barrier functions, we give in Figures 7.2 and 7.3 a series of contour and three-dimensional plots of the computed solutions and their gradients for three different boundary conditions. Figure 7.2 corresponds to a situation where $H=0, \delta \equiv 0$, and $\alpha \equiv 0$ in the top plate and bottom plate, respectively. In this case the solution has an oscillation in $x$ smaller than $2 \pi$. 

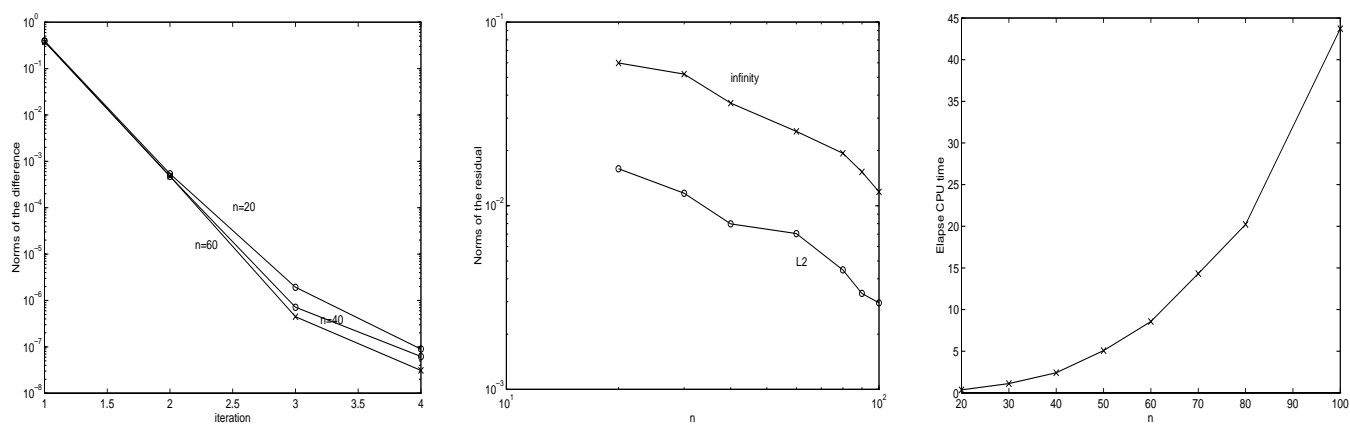

FIG. 7.1. Log-log plot of the infinity norm of the difference of two successive iterants of Newton method for discretization parameter $n=20,40,60$ versus the iteration number (left), semilog plot of the infinity and $L_{2}$ norms of the residual versus the discretization parameter $n$ (middle), and plot of the per-iteration CPU time versus the discretization parameter $n$ (right).
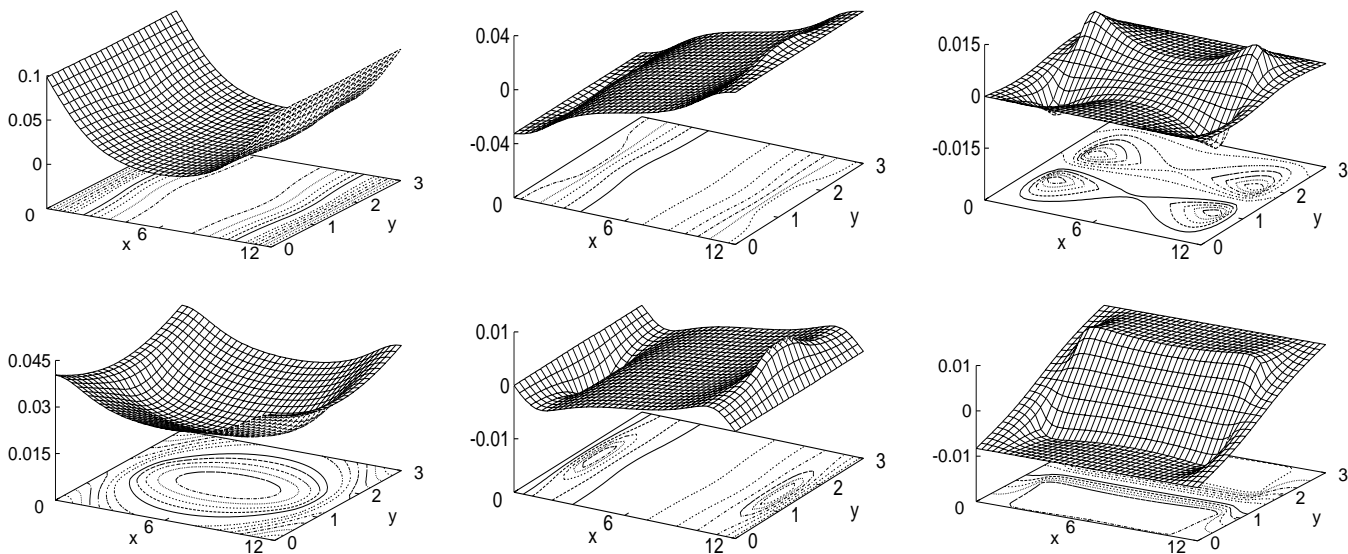

FIG. 7.2. Plots of the solution (left column) and the derivatives in the $x$ - (middle column) and the $y$ - (right column) directions for $H=0$ and $\alpha=.033, \delta=0$ (top) and $\alpha=0, \delta=.0083$ (bottom).
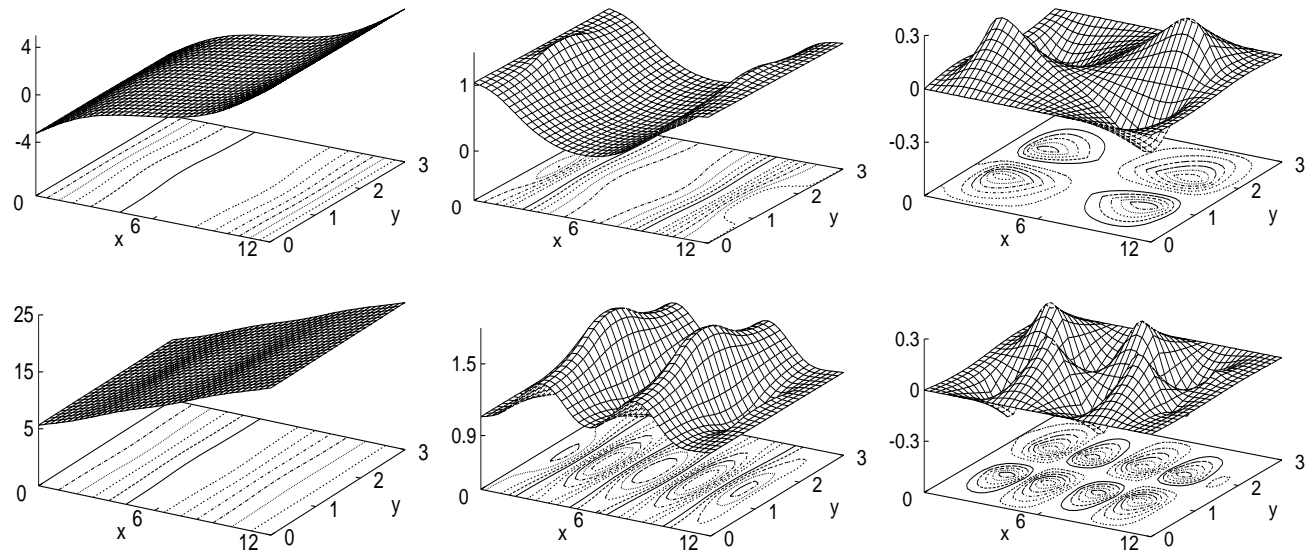

FIG. 7.3. Plots of the solution (left column) and the derivatives in the $x$ - (middle column) and the $y$ - (right column) directions for $H=1.1 \alpha=.05$ and $\delta=0$ for one-fluxon (top) and three-fluxon (bottom) solutions. 
TABLE 7.1

A priori bounds for the gradients of the solution associated with the PDE problems considered in Figures 7.2 and 7.3.

\begin{tabular}{||l||l|l||}
\hline Figures & Lemma 3.1 & Lemma 5.1 \\
\hline \hline 7.2 top & $\frac{\partial \phi}{\partial x} \leq 12+0.055(x-6)$ & $\frac{\partial \phi}{\partial x} \leq 0.448$ \\
& $\frac{\partial \phi}{\partial y} \leq 3$ & $\frac{\partial \phi}{\partial y} \leq 0.14$ \\
\hline \hline \multirow{2}{*}{7.2 bottom } & $\frac{\partial \phi}{\partial x} \leq 12$ & $\frac{\partial \phi}{\partial x} \leq 0.223$ \\
& $\frac{\partial \phi}{\partial y} \leq 3+0.00553 y$ & $\frac{\partial \phi}{\partial y} \leq 0.173$ \\
\hline \hline \multirow{2}{*}{7.3 top } & $\frac{\partial \phi}{\partial x} \leq 13.1+0.00833(x-6)$ & $\frac{\partial \phi}{\partial x} \leq 3.72$ \\
& $\frac{\partial \phi}{\partial y} \leq 3$ & $\frac{\partial \phi}{\partial y} \leq 0.447$ \\
\hline \hline \multirow{2}{*}{7.3 bottom } & $\frac{\partial \phi}{\partial x} \leq 13.1+0.055(x-6)$ & $\frac{\partial \phi}{\partial x} \leq 6.43$ \\
& $\frac{\partial \phi}{\partial y} \leq 3$ & $\frac{\partial \phi}{\partial y} \leq 0.447$ \\
\hline
\end{tabular}

For a larger value of the magnetic field $H=1.1$, shown in Figure 7.3, the oscillation in $x$ of the solution increases. The existence of more than one solution for certain values of the boundary conditions is confirmed here. The first solution presented in the top plate has an oscillation of less than $2 \pi$ (one-fluxon solution) while the oscillation of the second one is between $4 \pi$ and $6 \pi$ (three-fluxon solution).

For the PDE problems considered in Figures 7.2 and 7.3 we present in Table 7.1 the a priori estimates for the gradients of the solution theoretically obtained using Lemmas 3.1 and 5.1, respectively. The confirmation of these lemmas can be readily obtained by comparing the entries of the table with the associated plots in the figures. We also easily see the improvement of the estimates in the $x$-direction obtained in section 5. To confirm the theoretically obtained estimates in Lemma 5.2 of the solution (in the special case where it has a constant value on the line $x=0$ ) we have computed using this lemma the upper and lower bounds of $\phi$ for the problems considered in the top of Figure 7.2,

$$
5.967(x-6)+0.5(x-6)^{2} \leq \phi \leq-6.033(x-6)-0.5(x-6)^{2} \quad \text { for } 0 \leq x \leq 6
$$

and

$$
-5.967(x-6)+0.5(x-6)^{2} \leq \phi \leq 6.033(x-6)-0.5(x-6)^{2} \quad \text { for } 6 \leq x \leq 12,
$$

and in the top of Figure 7.3,

$$
5.95(x-6)+0.5(x-6)^{2} \leq \phi \leq-6.05(x-6)-0.5(x-6)^{2} \quad \text { for } 0 \leq x \leq 6
$$

and

$$
-5.95(x-6)+0.5(x-6)^{2} \leq \phi \leq 6.05(x-6)-0.5(x-6)^{2} \quad \text { for } 6 \leq x \leq 12 .
$$

As is easily seen these estimates agree with the numerical data presented in the associated figures.

As mentioned in section 2 an important question from both a theoretical and a practical point of view is, For what values of $H, \alpha$, and $\delta$ does the solution to our PDE problem exist? Or, equivalently, Which is the maximum current $I_{t}=2(\alpha \mathcal{W}+\delta \mathcal{L})$ that the device can carry for a given magnetic field? We have numerically determined 


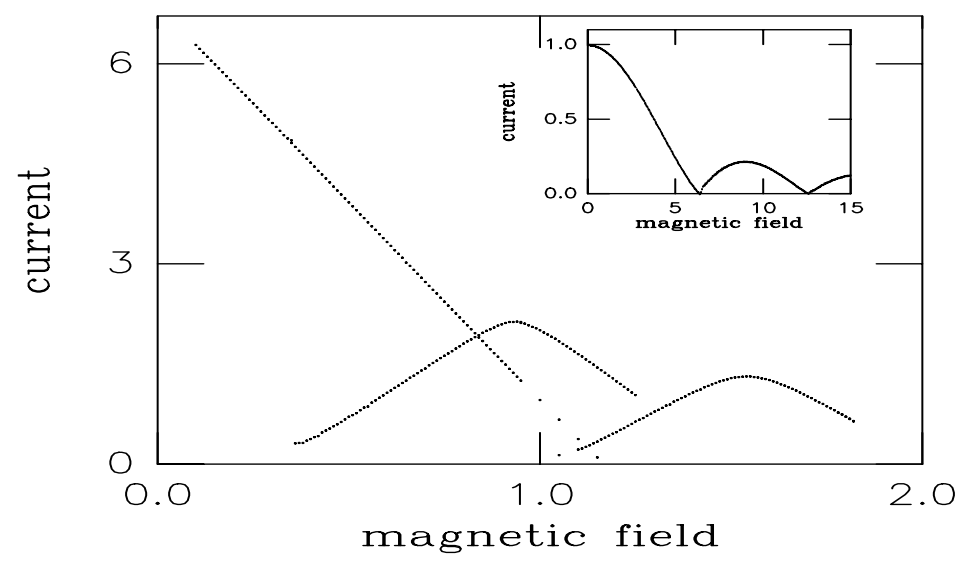

FIG. 7.4. Allowed values for magnetic field and current for the inline geometry $(\delta \equiv 0)$.

TABLE 7.2

Oscillations for the solutions corresponding to the branches of Figure 7.4 for several values of the magnetic field $H$.

\begin{tabular}{||l|l|l|l||}
\hline$H$ & $I_{t}$ & osc $_{x}$ & osc $_{y}$ \\
\hline \hline $10^{-2}$ & 6.65 & 3.73 & 0.21 \\
\hline 0.41 & 4.37 & 4.28 & 0.21 \\
\hline 0.47 & 0.52 & 7.75 & 0.21 \\
\hline 0.81 & 2.13 & 5.51 & 0.21 \\
\hline 1.01 & 1.97 & 11.54 & 0.20 \\
\hline 1.21 & 1.23 & 12.89 & 0.19 \\
\hline
\end{tabular}

the relation between the magnetic field and the maximum current for the case where $\delta=0$, and we present it graphically in Figure 7.4. It is important to note that for pairs of currents and magnetic fields below each (starting from the leftmost) of the three "maximum lines" shown, there exist one-fluxon, two-fluxon, and three-fluxon solutions, respectively. Above them no solutions exist. The overlap of the branches corresponding to one fluxon and three fluxons is consistent with the observation made from Figure 7.3 on the coexistence of a one-fluxon solution and three-fluxon solution for $H=1.1$. Notice also that in this case the maximum current which is obtained for $H=0$ is significantly lower than the bound given by (2.3), which is $l \times w=10$.

We have calculated the oscillations in the $x$ - and $y$-directions for the solutions corresponding to the maximum current for several values of the magnetic field and reported them in Table 7.2. An initial observation is that the oscillations in the $y$ direction are small and do not vary significantly as a function of $H$ for the values considered. This indicates that a one-dimensional description of this problem could be possible; such a heuristic approach based on an appropriate rescaling of a onedimensional sine-Gordon equation is currently under way. In turn the oscillations in the $x$-direction vary from $\pi$ to $6 \pi$ and correspond to the different fluxon branches described in the introduction. Notice, however, that the oscillation for the solution at the right-hand tip of the first branch is larger than $2 \pi$, contrary to what happens for the pure one-dimensional sine-Gordon equation. This is due to the fact that the 


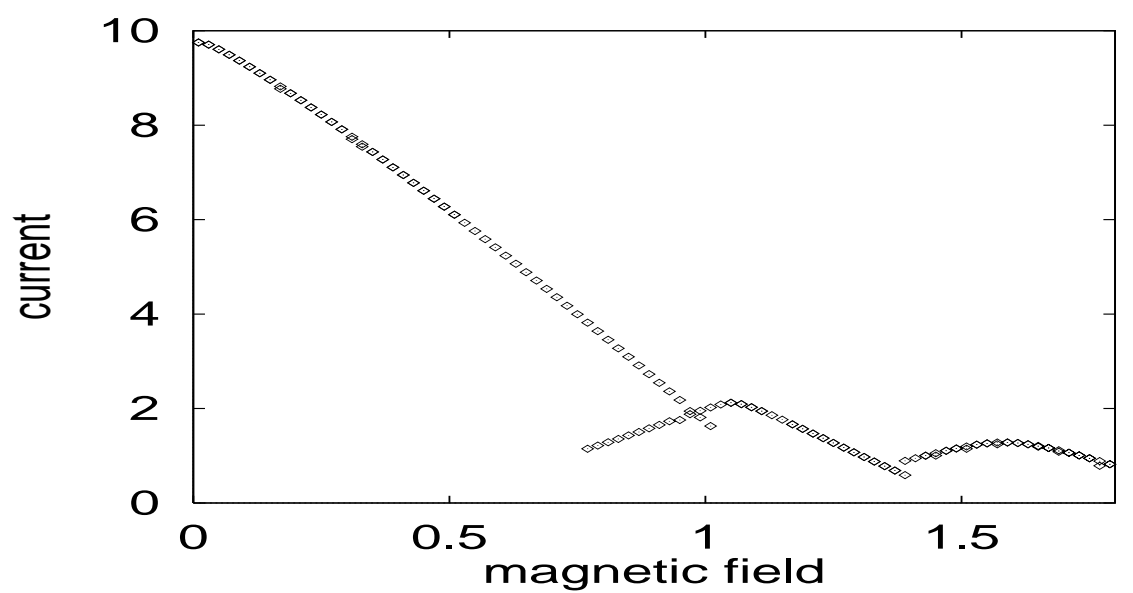

FIG. 7.5. Allowed values for magnetic field and current for the overlap geometry $(\alpha \equiv 0)$.

TABLE 7.3

Oscillations for the solutions corresponding to the branches of Figure 7.5 for several values of the magnetic field $H$.

\begin{tabular}{||l|l|l|l||}
\hline$H$ & $I_{t}$ & osc $_{x}$ & osc $_{y}$ \\
\hline \hline $10^{-2}$ & 9.75 & 0.91 & 0.54 \\
\hline 0.41 & 6.94 & 3.05 & 0.45 \\
\hline 0.81 & 3.45 & 5.43 & 0.33 \\
\hline 0.87 & 1.50 & 9.43 & 0.24 \\
\hline 1.11 & 1.94 & 11.87 & 0.27 \\
\hline 1.61 & 1.27 & 18.75 & 0.21 \\
\hline
\end{tabular}

junction domain is smaller than $\Omega$. The case of a very small domain $\Omega=[0,3] \times[0,3]$ and a window $\Omega_{j}=[1,2] \times[1,2]$ is presented in the inset of Figure 7.4. Another interesting feature is that the branches do not overlap and that their graph is very well approximated by $\left|\frac{\sin \frac{H}{2}}{\frac{H}{2}}\right|$, a feature that is well known for small Josephson junctions [2]. In this situation, the maximum current for $H=0$ is $l \times w=1$, corresponding to a solution equal to $\frac{\pi}{2}$ inside the junction.

Returning to the long junction, we have calculated the maximum current when the distribution is of the overlap type $(\alpha=0)$ and present it in Figure 7.5. In this case the maximum current for $H=0$ is very close to the theoretical bound $l \times w=10$, indicating that the solution inside the junction is very close to $\frac{\pi}{2}$. As in the inline case the branches overlap, indicating a multiplicity of solutions. The oscillations are reported in Table 7.3. Contrary to the inline case discussed above the oscillation in the $y$-direction varies significantly, indicating a stronger two-dimensional variation of the solution. As expected the values of the magnetic field corresponding to the zeros of the current coincide in Figures 7.4 and 7.5.

It is possible to use the bounds obtained on $\frac{\partial \phi}{\partial x}$ and $\frac{\partial \phi}{\partial y}$ in section 5 to obtain an estimate of the total current $I_{t}$. To do this notice by integrating the PDE (2.1)-(2.2) 
over the domains $\Omega_{j}$ and $\Omega$ that

$$
I_{t}=2(\alpha \mathcal{W}+\delta \mathcal{L})=\int_{\Omega_{j}} \sin \phi d x d y=\int_{\partial \Omega_{j}} \nabla \phi \mathbf{n} d s=\int_{\partial \Omega_{j}}\left(\frac{\partial \phi}{\partial x} \mathrm{n}_{\mathrm{x}}+\frac{\partial \phi}{\partial y} \mathrm{n}_{\mathrm{y}}\right) d s
$$

where the last integral is a flux integral taken on the boundary of the junction $\partial \Omega_{j}$, and $\mathbf{n}=\left(\begin{array}{l}n_{x} \\ n_{y}\end{array}\right)$ is the normal vector associated to this boundary. This integral can be bounded in the case of a junction with arbitrary shape and perimeter $P$,

$$
\left|\int_{\partial \Omega_{j}}\left(\frac{\partial \phi}{\partial x} \mathrm{n}_{\mathrm{x}}+\frac{\partial \phi}{\partial y} \mathrm{n}_{\mathrm{y}}\right) d s\right| \leq\left(\max \left|\frac{\partial \phi}{\partial x}\right|+\max \left|\frac{\partial \phi}{\partial y}\right|\right) P
$$

so that using Lemma 5.2 one obtains the following inequality for $\alpha$ and $\delta$ assumed positive:

$$
\frac{I_{t}}{2}=\alpha \mathcal{W}+\delta \mathcal{L} \leq \frac{P}{2}\left(\sqrt{2 o s c_{x}+(H+\alpha)^{2}}+\sqrt{2 o s c_{y}+\delta^{2}}\right) .
$$

In the case of a rectangular junction centered in the domain $\Omega$, this estimate can be improved by separating the integrals on the parts of the boundary $\partial \Omega_{j}$ parallel to the $x$ - and $y$-directions to obtain

$$
\begin{aligned}
\int_{\partial \Omega_{j}}\left(\frac{\partial \phi}{\partial x} \mathrm{n}_{\mathrm{x}}+\frac{\partial \phi}{\partial y} \mathrm{n}_{\mathrm{y}}\right) d s= & \int_{-\frac{l}{2}}^{\frac{l}{2}}\left[\frac{\partial \phi}{\partial y}\left(x, \frac{w}{2}\right)-\frac{\partial \phi}{\partial y}\left(x,-\frac{w}{2}\right)\right] d x \\
& +\int_{-\frac{w}{2}}^{\frac{w}{2}}\left[\frac{\partial \phi}{\partial x}\left(\frac{l}{2}, y\right)-\frac{\partial \phi}{\partial x}\left(-\frac{l}{2}, y\right)\right] d y
\end{aligned}
$$

Recall that $l$ and $w$ are the length and width of the junction domain $\Omega_{j}$, respectively. One can then bound the absolute values of the above integrals using Lemma 5.2 and obtain

$$
\frac{I_{t}}{2}=\alpha \mathcal{W}+\delta \mathcal{L} \leq \sqrt{2 o s c_{x}+(H+\alpha)^{2}} w+\sqrt{2 o s c_{y}+\delta^{2}} l
$$

This upper bound for the current is not as sharp as (2.3). For example, for the case of Figure 7.4 for $H=0.41$ we find $I_{t}=4.37$ corresponding to $\alpha=0.728$. Using the values of the oscillations given by Table 7.2 we obtain for the right-hand side of the inequality (7.3) 9.62, which corresponds to a total current of 19.24, while the maximum current allowed is $l \times w=10$.

8. Conclusions and future work. Josephson junctions have already proved themselves to be technologically useful, and it is our belief that their importance will increase significantly in the near future. Many recent reports and books have been dedicated to the analysis of the one-dimensional case, where one can usually give the solution of the associated boundary value problem analytically in terms of elliptic functions. Our report is, to the best of our knowledge, the first to try to theoretically analyze the semilinear PDE problem that effectively and accurately models two-dimensional window Josephson junctions. Specifically we established the existence of solutions and obtained regularity and a priori estimates for the derivatives of the solution. We had to use a specific method to establish these estimates instead of the well-known theory for elliptic PDEs [9] because the solution is defined modulo a 
multiple of $2 \pi$ due to the periodicity of the nonlinearity and the Neumann boundary conditions, and therefore its norm cannot be bounded.

From the practical point of view this study validates the practical observation that for a pure junction $\left(\Omega_{j} \equiv \Omega\right)$ of small dimensions $\mathcal{L}<2, \mathcal{W}<2$ and zero boundary conditions the only solutions are the constants $n \pi$, where $n$ is an integer. Another important practical result is that if $I_{j}$ depends only on $x$ and $\delta \equiv 0$ and if $\mathcal{W}<2$, then $\frac{\partial \phi}{\partial y} \equiv 0$. It is interesting to notice that in both results, the value 2 comes up. The same value appears in the one-dimensional reductions of the problem, where it corresponds to the maximum of $\frac{\partial \phi}{\partial x}$ for the separatrix of the pendulum phase space.

The theoretical analysis of two-dimensional window Josephson junctions is by no means complete. Below are some of the issues that are of practical interest (and as such some experimental analysis has already been carried out), and their theoretical analysis will be mathematically challenging.

Notice that all a priori estimates obtained are independent of the window $\Omega_{j}$. This is due to the fact that we bound $|\sin (\phi)|$ by 1 very early in our analysis. Therefore, although these estimates seem to be very generous for the PDE problems considered in Table 7.1, they are sharp for large windows. Nevertheless, since many important physical properties of Josephson junctions depend on the size and the geometry of the window $[4,5]$ new a priori estimates which sense the geometrical parameters of the window would be of importance.

The maximum current that a Josephson junction can carry for a given configuration of $\alpha$ 's and $\delta$ 's and for a given $H$ is another point of interest, and its theoretical estimation is a challenging and difficult problem. One approach for that is carrying out a three-parameter stability analysis. These parameters are the values at the boundary conditions and the size of the window. Such stability analysis to determine the turning and bifurcation points and eigenvalues corresponding to the different solutions is under way.

The method of Newton proved to be a very reliable and efficient linearization tool. We believe that the proof of its quadratic convergence at continuum level (PDE analysis) or discrete level (numerical analysis) is another interesting mathematical problem. This problem does not have a unique solution and is therefore ill-posed in the Hadamard sense.

Appendix. Proof of existence of the generalized solution for the auxiliary problem. To apply the Leray-Schauder theorem we consider the Banach space $\mathcal{B}$,

$$
\mathcal{B}=\left\{u \in C^{1}(\bar{\Omega}) \text { and } \int_{\Omega} u d x d y=0\right\}
$$

and construct the mapping

$$
\kappa T: \forall u \in \mathcal{B} \longrightarrow w
$$

where $w$ is the solution of the problem

$$
\begin{gathered}
\Delta w=\kappa\left[\mathcal{I}_{j} \sin (u+\zeta+f)-\frac{1}{\mu(\Omega)} \int_{\Omega_{j}} \sin (u+\zeta+f) d x d y\right] \equiv \mathcal{F}(x, y) \quad \text { in } \Omega, \\
\frac{\partial w}{\partial n}=0 \quad \text { on } \partial \Omega
\end{gathered}
$$


Note that we have formed the right-hand side $\mathcal{F}$ so that the solution of the above linear PDE problem exists up to a constant and, as it can be easily seen using the a priori estimates obtained in section 3 , it belongs to $C^{1, \gamma}(\bar{\Omega})$. From this class of infinitely many solutions, we can select (by choosing the appropriate constant) the one that satisfies relation (4.7). Therefore we have constructed a mapping from $\mathcal{B}$ to $\mathcal{B}^{\gamma}$, where $\mathcal{B}^{\gamma}=\left\{u \in C^{1, \gamma}(\bar{\Omega})\right.$ and $\left.\int_{\Omega} u d x d y=0\right\}$. The mapping $T: \mathcal{B} \longrightarrow \mathcal{B}^{\gamma}$ is bounded and hence $T: \mathcal{B} \longrightarrow \mathcal{B}$ is compact. To apply the Leray-Schauder theorem, and therefore to prove the existence of a fixed point of $T$, we need only show that for every solution of $w=\kappa T w, \kappa \in[0,1]$ we have that $\|w\|_{C^{1}(\bar{\Omega})}$ is bounded. This is a direct consequence of the a priori estimates we have already obtained. Note that from Schauder estimates we also have that the above-mentioned solution belongs to $C^{3}(\Omega)$.

\section{REFERENCES}

[1] A. Barone, F. Esposito, K. Likharev, V. Semenov, B. Todorov, and R. Vaglio, Effect of boundary conditions upon the phase in two-dimensional Josephson junctions, J. Appl. Phys., 53 (1982), pp. 5802-5809.

[2] A. Barone and G. Paterno, Physics and Applications of the Josephson Effect, John Wiley, New York, 1982.

[3] J. Caputo, N. Flytzanis, Y. Gaididei, and E. Vavalis, Two-dimensional effects in Josephson junctions: Static properties, Phys. Rev., (1996), pp. 2092-2021.

[4] J. Caputo, N. Flytzanis, and E. Vavalis, A semi-linear elliptic PDE model for static solution of Josephson junctions, Internat. J. Modern Phys. C, 6 (1995), pp. 241-262.

[5] J. Caputo, N. Flytzanis, And E. Vavalis, Effect of geometry on fluxon width in a Josephson junction, Internat. J. Modern Phys. C, 7 (1996), pp. 191-216.

[6] J. G. Caputo, N. Flytzanis, and M. Devoret, Dressed fluxon in a window Josephson junction, Phys. Rev. B, 50 (1994), pp. 6471-6474.

[7] T. CHOw, Enhancement of the critical current in a Josephson tunneling junction with defect size in micrometers, Phys. C, 255 (1995), pp. 311-318.

[8] R. Flesch, M. Forest, AND A. Sinha, Numerical inverse spectral transform for periodic sine-Gordon equation: Theta function solutions and their linearized stability, Phys. D, 48 (1991), pp. 169-231.

[9] D. Gilbarg And N. Trudinger, Elliptic Partial Differential Equations of Second Order, Springer-Verlag, New York, 1983.

[10] E. P. Houmman, J. G. Gijsbersten, J. Flokstra, and H. Rogalla, On the suppression of the sidelobes of the supercurrent in small Josephson, Phys. C, 164 (1991), pp. 339-344.

[11] S. KRuzhKov, Quasilinear parabolic equations and systems with two independent variables, Trudy Sem. Petrovsk Vyp., 5 (1979), pp. 217-272.

[12] O. A. Ladyzhenskaya, The Boundary Value Problems of Mathematical Physics, Prentice-Hall, Englewood Cliffs, NJ, 1986.

[13] O. A. Ladyzhenskaya and N. N. Uraltseva, Linear and Quasilinear Elliptic Equations, Academic Press, New York, 1968.

[14] J. Ortega and W. Rheinboldt, Iterative Solution of Nonlinear Equations in Several Variables, Academic Press, New York, 1970.

[15] C. Owen and D. Scalapino, Vortex structure and critical currents in Josephson junctions, Phys. Rev., 164 (1967), pp. 538-544.

[16] J. Rice And R. Boisvert, Solving Elliptic Problems Using ELLPACK, Springer-Verlag, New York, 1985.

[17] A. Tersenov, On the first boundary value problem for quasilinear parabolic equations with two independent variables, Arch. Ration. Mech. Anal., 152 (2000), pp. 81-92.

[18] A. Tersenov, On quasilinear non-uniformly parabolic equations in general form, J. Differential Equations, 142 (1998), pp. 263-276. 